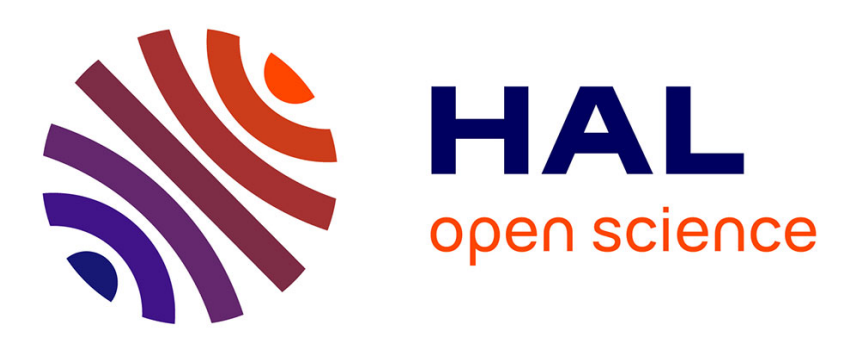

\title{
A hybrid model of opinion dynamics with memory-based connectivity
}

\author{
Simone Mariano, Irinel-Constantin Morarescu, Romain Postoyan, Luca
}

Zaccarian

\section{- To cite this version:}

Simone Mariano, Irinel-Constantin Morarescu, Romain Postoyan, Luca Zaccarian. A hybrid model of opinion dynamics with memory-based connectivity. IEEE Control Systems Letters, 2020, 4 (3), pp.644-649. 10.1109/LCSYS.2020.2989077 . hal-02545383

\section{HAL Id: hal-02545383 \\ https://hal.science/hal-02545383}

Submitted on 17 Apr 2020

HAL is a multi-disciplinary open access archive for the deposit and dissemination of scientific research documents, whether they are published or not. The documents may come from teaching and research institutions in France or abroad, or from public or private research centers.
L'archive ouverte pluridisciplinaire HAL, est destinée au dépôt et à la diffusion de documents scientifiques de niveau recherche, publiés ou non, émanant des établissements d'enseignement et de recherche français ou étrangers, des laboratoires publics ou privés. 


\title{
A hybrid model of opinion dynamics with memory-based connectivity
}

\author{
S. Mariano, I.C. Morărescu, R. Postoyan Member, IEEE, L. Zaccarian Fellow, IEEE
}

\begin{abstract}
Given a social network where the individuals know the identity of the other members, we present a model of opinion dynamics where the connectivity among the individuals depends on both their current and past opinions. Thus, their interactions are not only based on the present states but also on their past relationships. The model is a multi-agent system with active or inactive pairwise interactions depending on auxiliary state variables filtering the instantaneous opinions, thereby taking the past experience into account. When an interaction is (de)activated, a jump occurs, leading to a hybrid model. The proven stability properties ensure that opinions converge to local agreements/clusters as time grows. Simulation results are provided to illustrate the theoretical guarantees.
\end{abstract}

Index Terms-Agents-based systems; Stability of hybrid systems; Lyapunov methods; Opinion Dynamics.

\section{INTRODUCTION}

$\mathbf{M}$ OTIVATED by the growing importance of digital social networks, opinion dynamics has received an increasing attention from the control community e.g., [1], [4], [18], [20], [22]. The multi-agent systems formalism is well-suited for modelling these networks, as a node can model the individual's opinion and an edge describes the interaction between two given individuals e.g., [5], [8], [14], [19].

Two main models provide convergence towards local agreement or disagreement patterns. One of them (FJ) [13] essentially filters the consensus dynamics by using the initial opinions. Indeed, a major role in the mutual influence on the individual's opinions is played by their culture, belonging to a community (social class, political party, etc), principles and beliefs. The second one is the bounded confidence model (HK) described in [17], which formalizes the idea that only individuals with similar opinions actually interact. Social psychologists agree that both the FJ and HK models are relevant, depending on the context, see [10] for a detailed survey. Nevertheless, as pointed out in [10], opinion dynamics in social networks is a complex phenomenon, whose key features cannot be completely captured by any of these models separately. This has motivated the development of other deterministic models e.g., [1], [5], [11], [20], [22], as well as stochastic models e.g., [2], [7], [19], [27]. Notably, most of these works provide either empirical or rigorous convergence results but stability is eluded in general. The recent work in [12] provides a Lyapunov

Work supported by the ANR under grant HANDY ANR-18-CE40-0010. S. Mariano, I.C. Morărescu and R. Postoyan are with the Université de Lorraine, CNRS, CRAN, F-54000 Nancy, France (e-mails: firstname.name@univlorraine.fr).

L. Zaccarian is with LAAS-CNRS, Université de Toulouse, CNRS, Toulouse, France and the Department of Industrial Engineering, University of Trento, Trento, Italy (e-mail: zaccarian@laas.fr). analysis of the HK model and reveals that only an attractivity property can be guaranteed. Then [12] proposes a stability inducing variant of the HK model where the connectivity depends on adaptive thresholds, instead of fixed ones as in [17]. Indeed, a link is activated when the opinions mismatch between two agents is small compared to the average opinion mismatch with their other neighbours.

This paper is a further step in the direction paved by [12]: we propose a model where connectivity also depends on the past history. With this, we merge the features of the FJ model (where importance is given to the past, the initial conditions) and those of the HK model (the bounded confidence mechanism based on current opinions). It is indeed reasonable to assume that the interactions within a social network depend both on the current state and on the past relationships, when the members are aware of the identity of their neighbours. Here we account for the past by linearly filtering the instantaneous (de)activation functions (de)activating a link only when both the adaptive threshold and its filtered version reach certain thresholds. Our model uses the hybrid formalism of [16]. A new Lyapunov function is constructed, which guarantees a suitable $\mathcal{K} \mathcal{L}$-stability property ensuring asymptotic convergence to opinion clusters. In addition, solutions are proved not to generate Zeno phenomenon and to stop jumping in finitetime. Simulation results illustrate the behaviour of the model and the impact of the filters and their parameters.

Our technical proofs exploit interesting analogies between the adaptive threshold connectivity as in [12] and the eventtriggered control technique of [25]. These two domains - a priori unrelated - have actually much in common. Indeed, the memory-based connectivity proposed here is inspired by the dynamic event-triggering control policy proposed in [15].

Background and problem statement are given in Section II. The new hybrid model and its stability analysis, are presented in Section III. Illustrative simulations results are reported in Section IV and Section V concludes the paper.

Notation. $\mathbb{R}$ represents the real numbers, $\mathbb{R}_{\geq 0}:=[0, \infty)$, $\mathbb{R}_{>0}:=(0, \infty) .|x|$ is the Euclidean norm of vector $x \in \mathbb{R}^{n}$. Moreover, $(x, y)$ stands for $\left[x^{\top} y^{\top}\right]^{\top}$. A continuous function $\beta: \mathbb{R}_{\geq 0} \times \mathbb{R}_{\geq 0} \longrightarrow \mathbb{R}_{\geq 0}$ is of class- $\mathcal{K} \mathcal{L}(\beta \in \mathcal{K} \mathcal{L})$, if it is non-decreasing in its first argument, non-increasing in its second argument, $\lim _{r \rightarrow 0^{+}} \beta(r, s)=0$ for each $s \in \mathbb{R}_{\geq 0}$, and $\lim _{s \rightarrow \infty} \beta(r, s)=0$ for each $r \in \mathbb{R}_{\geq 0}$. $U^{\circ}(x ; v):=$ $\limsup _{h \rightarrow 0^{+}, y \rightarrow x}(U(y+h v)-U(y)) / h$ denotes the Clarke generalized directional derivative at $x$ in the direction $v$ of a Lipschitz function $U$ [6]. 


\section{BACKGROUND AND PROBLEM STATEMENT}

Following [12], we consider a set of individuals $\mathcal{V}:=$ $\{1, \ldots, n\}$, also referred to as agents, connected through a social network. The opinions of individuals are modelled by a scalar variable $y_{i} \in \mathbb{R}$ for any $i \in \mathcal{V}$. The dynamics of opinion $y_{i}, i \in \mathcal{V}$, depends on the interactions of individual $i$ with its neighbours. We define

$$
\mathcal{E}^{+}:=\{(i, j) \in \mathcal{V} \times \mathcal{V} \mid i<j\}
$$

and, for each $(i, j) \in \mathcal{E}^{+}$, adjacency coefficient $a_{i j}$ defines whether agents $i$ and $j$ interact or not, i.e. whether or not they are neighbours. Thus, $a_{i j}$ is the connectivity variable for link $(i, j)$, satisfying

$$
a_{i j}=a_{j i}:= \begin{cases}1 & \text { if } i \text { and } j \text { interact } \\ 0 & \text { otherwise. }\end{cases}
$$

The graph is undirected as $a_{i j}=a_{j i}$. Interaction changes, if any, are described by a jump of the variable $a_{i j}$. The corresponding hybrid behavior is well represented with the formalism of [16]. Variables $y_{i}$ and $a_{i j}$ obey the next continuoustime dynamics between two successive jumps

$$
\begin{aligned}
\dot{y}_{i} & =\sum_{j=1}^{n} \varphi_{i j}\left(y_{j}-y_{i}\right), & & \forall i \in \mathcal{V}, \\
\dot{a}_{i j} & =0, & & \forall(i, j) \in \mathcal{E}^{+},
\end{aligned}
$$

with $\varphi_{i j}:=\frac{a_{i j}}{d_{i} d_{j}}$ when $i \neq j$ and $\varphi_{i i}:=-\sum_{j \neq i} \varphi_{i j}$, where $d_{i}:=1+\sum_{j \neq i} a_{i j} \geq 1$ is the degree of agent $i$ augmented by 1 . We omit the dependence of $d_{i}$ and $\varphi_{i j}$ on the connectivity variables. By construction we have that $\varphi_{i j}=\varphi_{j i}$ and $\sum_{j=1}^{n} \varphi_{i j}=0$ for any $i \in \mathcal{V}$. The variable $\varphi_{i j}$ is such that $\Phi:=\left[-\varphi_{i j}\right]_{(i, j) \in \mathcal{V}^{2}}$ defines a normalized Laplacian matrix. Dynamics (4) means that $a_{i j}$ is constant between jumps (along flowing solutions) and that the time-derivative of $y_{i}$ is given by the weighted average of the opinion mismatch between agent $i$ and its neighbours.

When a jump occurs over the network, i.e. when one of the variables $a_{i j}$ for some $(i, j) \in \mathcal{E}^{+}$is updated, solutions obey the following discrete dynamics,

$$
\begin{array}{ll}
y_{h}^{+}=y_{h}, & \forall h \in \mathcal{V} \\
a_{h k}^{+}=\left\{\begin{array}{ll}
a_{h k} & \text { if }(h, k) \neq(i, j) \\
1-a_{h k} & \text { if }(h, k)=(i, j),
\end{array} \quad \forall(h, k) \in \mathcal{E}^{+}\right.
\end{array}
$$

Dynamics (5) states that the opinions $y_{i}$ do not change across jumps and that the connectivity variable $a_{i j}$ toggles between 0 and 1 according to (de)activation. It simplifies notation to write the second equation of system (5) as

$$
a^{+}=g_{i j}(y, a),
$$

where $y:=\left(y_{1}, \ldots, y_{n}\right) \in \mathbb{R}^{n}$ is the opinions vector, and $a:=\left(a_{12}, \ldots, a_{1 n}, a_{23}, \ldots, a_{n-2, n}, a_{n-1, n}\right) \in\{0,1\}^{\frac{n(n-1)}{2}}$ is the connectivity variables vector.

To complete the model, we present the memoryless (de)activation criterion (jump dynamics) for each link between two agents, as proposed in $[12, \S 4]$. The adaptive thresholds idea of [12] is that two agents interact when their opinions are close relative to their respective neighbours' opinions (an alternative to the fixed threshold HK model [17]). Roughly speaking, given $(i, j) \in \mathcal{E}^{+}$:

- Deactivation. If $a_{i j}=1, \operatorname{link}(i, j)$ is active. Deactivation is then enabled when $\Gamma_{i j}^{\mathrm{off}}(y, a) \leq-\varepsilon$, where $\varepsilon>0$ is a regularization parameter and $\eta>0$ is a connectivity parameter, while $\Gamma_{i j}^{\text {off }}$ is defined in (7) at the top of the next page. This means that link $(i, j)$ is cut when $y_{i}$ and $y_{j}$ are too far apart, as compared to other neighbours' opinions. Parameter ${ }^{1} \varepsilon>0$ rules out Zeno solutions, i.e. solutions that jump indefinitely in a finite continuous time interval. It is typically set to a small value.

- Activation. If $a_{i j}=0$, link $(i, j)$ is not active. Activation is enabled when $\Gamma_{i j}^{\mathrm{on}}(y, a) \geq \varepsilon$ with $\Gamma_{i j}^{\mathrm{on}}$ defined in (7). The underlying idea is that link $(i, j)$ should be activated when the difference between opinions $i$ and $j$, namely $\left|y_{i}-y_{j}\right|$ is small as compared to the average opinion mismatch of agents $i$ and $j$ with their respective neighbours (individuals with relatively close opinions influence each other).

Parameter $\eta$, as empirically shown in [12], influences the average cardinality of the communities that arise from the initial topology. It actually determines how big the mismatch $\left|y_{i}-y_{j}\right|$ needs to be with respect to the average opinions mismatch of agents $i$ and $j$ with their neighbours to (de)activate the link.

As a result, the overall hybrid model is given by

$$
\begin{aligned}
& {\left[\begin{array}{c}
\dot{y} \\
\dot{a}
\end{array}\right]=\left[\begin{array}{c}
-\Phi y \\
0
\end{array}\right], \quad(y, a) \in C_{\text {inst }}} \\
& {\left[\begin{array}{c}
y^{+} \\
a^{+}
\end{array}\right] \in\left[\bigcup_{\substack{(y, a) \in D_{i j, \text { inst }} \\
(i, j) \in \mathcal{E}^{+}}}^{y} g_{i j}(y, a)\right], \quad(y, a) \in D_{\text {inst }},}
\end{aligned}
$$

where we recall that $\Phi=\left[-\varphi_{i j}\right]_{(i, j) \in \mathcal{V}^{2}}$ and $\mathcal{E}^{+}$is in (1), and $\mathbb{X}_{\text {inst }}:=\mathbb{R}^{n} \times\{0,1\}^{\frac{n(n-1)}{2}}$, and

$$
\begin{aligned}
& D_{i j, \text { inst }}^{\text {on }}:=\left\{(y, a) \in \mathbb{X}_{\text {inst }} \mid a_{i j}=0, \Gamma_{i j}^{\text {on }}(y, a) \geq \varepsilon\right\} \\
& D_{i j, \text { inst }}^{\text {off }}:=\left\{(y, a) \in \mathbb{X}_{\text {inst }} \mid a_{i j}=1, \Gamma_{i j}^{\text {off }}(y, a) \leq-\varepsilon\right\},
\end{aligned}
$$

$D_{\text {inst }}:=\bigcup_{(i, j) \in \mathcal{E}^{+}} D_{i j \text {,inst }}^{\text {on }} \cup D_{i j \text {,inst }}^{\text {off }}$, and $C_{\text {inst }}:=\overline{\mathbb{X}_{\text {inst }} \backslash D_{\text {inst }}}$. The main stability result of [12] is to prove that all maximal solutions (solutions that cannot be extended any further in time) to (7), (8) are complete (solutions that evolve for arbitrarily large times) and eventually continuous (i.e, they perform a finite number of jumps), see [16, Chapter 2], and all enjoy a desirable global asymptotic stability property for the following set $\mathcal{A}_{\text {inst }}$, measured by the function $\omega_{0}$ below,

$$
\begin{aligned}
\mathcal{A}_{\text {inst }} & :=\left\{(y, a) \in \mathbb{X}_{\text {inst }} \mid a_{i j}\left(y_{i}-y_{j}\right)^{2}=0, \forall(i, j) \in \mathcal{E}^{+}\right\}, \\
\omega_{0}(y, a) & :=\min _{(z, a) \in \mathcal{A}_{\text {inst }}}|y-z| .
\end{aligned}
$$

Set $\mathcal{A}_{\text {inst }}$ characterizes clusters as, when $(y, a) \in \mathcal{A}_{\text {inst }}$, any couple of agents $(i, j)$ either have the same opinion, namely $y_{i}=y_{j}$, which means they belong to the same cluster, or they do not share any interconnection, so that $a_{i j}=0$. Since $\omega_{0}$ is not a Euclidean norm in the extended $(y, a)$ space (because $a$ is fixed when defining $\omega_{0}$ in (9)), we deem it more appropriate

\footnotetext{
${ }^{1}$ Constant $\varepsilon$ is the same for every link of the network in [12], however the results do hold mutatis mutandis when it is link dependent, i.e. when we have different $\varepsilon_{i j}>0$ for each $(i, j) \in \mathcal{V}^{2}$.
} 


$$
\begin{aligned}
\Gamma_{i j}^{\mathrm{on}}(y, a) & :=\sum_{\ell \neq i, \ell \neq j}\left[\left(d_{j}+1\right) \varphi_{i \ell}\left(y_{i}-y_{\ell}\right)^{2}+\left(d_{i}+1\right) \varphi_{j \ell}\left(y_{j}-y_{\ell}\right)^{2}\right]-\left(1+\frac{\eta^{2}}{d_{i} d_{j}}\right)\left(y_{i}-y_{j}\right)^{2} \\
\Gamma_{i j}^{\mathrm{off}}(y, a) & :=\sum_{\ell \neq i, \ell \neq j}\left[\frac{d_{j} a_{i \ell}}{\left(d_{i}-1\right) d_{\ell}}\left(y_{i}-y_{\ell}\right)^{2}+\frac{d_{i} a_{j \ell}}{\left(d_{j}-1\right) d_{\ell}}\left(y_{j}-y_{\ell}\right)^{2}\right]-\left(1-\frac{\eta^{2}}{d_{i} d_{j}}\right)\left(y_{i}-y_{j}\right)^{2}
\end{aligned}
$$

to use in this paper the following notion of $\mathcal{K} \mathcal{L}$-stability, which combines the approach in [26] with the $\mathcal{K} \mathcal{L}$ results in [3, §3.5]. Following [12], the solutions of hybrid model (8) evolve in two time directions, on of them, $t$, keeping track of ordinary time and a second one, $\mathfrak{j}$, counting the number of jumps.

Definition 1: Let $\omega: \mathbb{R}^{n_{q}} \rightarrow \mathbb{R}_{\geq 0}$ be continuous. A hybrid system is $\mathcal{K} \mathcal{L}$-stable with respect to $\omega$ if there exists $\beta \in \mathcal{K} \mathcal{L}$ such that all maximal solutions $\phi$ are complete and satisfy $\omega(\phi(\mathfrak{t}, \mathfrak{j})) \leq \beta(\omega(\phi(0,0)), \mathfrak{t}+\mathfrak{j})$ for all $(\mathfrak{t}, \mathfrak{j}) \in \operatorname{dom} \phi$.

It is proven in the text beneath [12, Lemma 5] that system (8) is $\mathcal{K} \mathcal{L}$-stable with respect to $\omega_{0}$. Due to the structure of $\mathcal{A}_{\text {inst }}$ where $a_{i j}\left(y_{i}-y_{j}\right)^{2}=0$, this property means that solutions asymptotically form clusters [12, Section 4.3].

A possible criticism of the result of [12] summarized above is that the connectivity variables $a_{i j}$ are only based on the instantaneous opinions mismatch, see (8b). If two agents had or had not been in agreement for a long time, their current interaction status is not affected by the past. The main contribution of this paper is to introduce a novel model with memory-based connectivity features. In the next section, we formalize this intuition via a new hybrid model where the past memory is captured by additional state variables. For this model, we will prove a generalization of the above mentioned $\mathcal{K} \mathcal{L}$-stability property.

\section{Memory-BASed CONNeCtivity}

\section{A. Hybrid model}

We define the connectivity between agents $i$ and $j$, for $(i, j) \in \mathcal{E}^{+}$, using $\Gamma_{i j}^{\text {on }}$ or $\Gamma_{i j}^{\text {off }}$ in (7), but also based on a new memory state variable $\theta_{i j} \in \mathbb{R}$ that is a filtered version of the instantaneous threshold criterion reviewed in Section II. Loosely speaking, $\theta_{i j}$ reflects the history of the interaction between agents $i$ and $j$.

More precisely, for each $(i, j) \in \mathcal{E}^{+}$, the flow dynamics for $\theta_{i j}$ is selected as

$$
\begin{aligned}
\dot{\theta}_{i j} & =-\beta_{i j} \theta_{i j}+\left(1-a_{i j}\right) \Gamma_{i j}^{\mathrm{on}}(y, a)+a_{i j} \Gamma_{i j}^{\mathrm{off}}(y, a) \\
& =: f_{\theta, i j}\left(y, a, \theta_{i j}\right),
\end{aligned}
$$

where $\beta_{i j}>0$ are tunable parameters associated to how fast each agent "forgets" the past, and $\Gamma_{i j}^{\text {on }}$ and $\Gamma_{i j}^{\text {off }}$ are given in (7). When link $(i, j)$ is active, $a_{i j}=1$ according to (2) and $\dot{\theta}_{i j}=-\beta_{i j} \theta_{i j}+\Gamma_{i j}^{\text {off }}(y, a)$ in view of (10). Hence, variable $\theta_{i j}$ filters $\Gamma_{i j}^{\text {off }}(y, a)$, which is indeed the right term to be monitored for deciding whether link $(i, j)$ should be deactivated, see Section II. Conversely, when link $(i, j)$ is not active, $a_{i j}=0$ and $\dot{\theta}_{i j}=-\beta_{i j} \theta_{i j}+\Gamma_{i j}^{\text {on }}(y, a)$ so that $\Gamma_{i j}^{\text {on }}(y, a)$ is filtered to infer whether or not the link should be activated.

The novel variable $\theta_{i j}$ acts as a first order filter for the thresholds $\Gamma_{i j}^{\text {off }}$ or $\Gamma_{i j}^{\text {on }}$, as shown in (10). Hence, $\beta_{i j}$ are tuning parameters to set how "nostalgic" each pair of agents are with respect to their common past. When $\beta_{i j}$ is large, the past is not given much credit, and $\beta_{i j} \rightarrow \infty$ recovers the criterion of Section II. Conversely, when $\beta_{i j}$ is small, the past values of $\Gamma_{i j}^{\text {off }}$ or $\Gamma_{i j}^{\text {on }}$ matter more, as compared to the current ones.

When a jump occurs, i.e. when a link is (de)activated, the memory variable $\theta_{i j}$ is unchanged, namely $\theta_{i j}^{+}=\theta_{i j}$ for each $(i, j) \in \mathcal{E}^{+}$. The proposed memory-based (de)activation policy then intuitively generalizes the one of Section II:

- Activation. If $a_{i j}=0$, link $(i, j)$ is not active. Activation is enabled when $\Gamma_{i j}^{\text {on }}(y, a) \geq \varepsilon$ and $\theta_{i j}$ is non-negative. Parameter $\varepsilon$ plays the same role as in Section II, preventing Zeno solutions, see footnote 1 on page 2 .

- Deactivation. If $a_{i j}=1$, link $(i, j)$ is active. Deactivation is then enabled when $\Gamma_{i j}^{\text {off }}(y, a) \leq-\varepsilon$ and $\theta_{i j}$ is non-positive. The rationale is similar to the previous case.

The mechanism described above can be written in a compact form extending the memoryless model (8). Introducing

$$
\begin{aligned}
& \theta:=\left(\theta_{12}, \ldots, \theta_{1 n}, \theta_{23}, \ldots, \theta_{n-2, n}, \theta_{n-1, n}\right) \in \mathbb{R}^{\frac{n(n-1)}{2}} \\
& x:=(y, a, \theta) \in \mathbb{X}_{\mathrm{mem}}:=\mathbb{R}^{n} \times\{0,1\}^{\frac{n(n-1)}{2}} \times \mathbb{R}^{\frac{n(n-1)}{2}},
\end{aligned}
$$

the memory-based hybrid model is given by

$$
\begin{aligned}
& {\left[\begin{array}{c}
\dot{y} \\
\dot{a} \\
\dot{\theta}
\end{array}\right]=f(x):=\left[\begin{array}{c}
-\Phi y \\
0 \\
f_{\theta}(y, a, \theta)
\end{array}\right],} \\
& {\left[\begin{array}{c}
y^{+} \\
a^{+} \\
\theta^{+}
\end{array}\right] \in g(x):=\left[\begin{array}{c}
y \\
\bigcup_{(y, a) \in D_{i j, \text { mem }}}^{(i, j) \in \mathcal{E}^{+}} \\
\theta
\end{array}\right], \quad x \in D_{\mathrm{mem}},}
\end{aligned}
$$

with $x:=(y, a, \theta)$, with

$$
\begin{aligned}
& D_{\text {mem }}:=\bigcup_{(i, j) \in \mathcal{E}^{+}} D_{i j, \mathrm{mem}}^{\mathrm{on}} \cup D_{i j, \mathrm{mem}}^{\mathrm{off}}, \quad C_{\mathrm{mem}}:=\overline{\mathbb{X}_{\mathrm{mem}} \backslash D_{\mathrm{mem}}} \\
& D_{i j \text {,mem }}^{\text {on }}:=\left\{(y, a, \theta) \in \mathbb{X}_{\mathrm{mem}} \mid a_{i j}=0, \Gamma_{i j}^{\mathrm{on}} \geq \varepsilon, \theta_{i j} \geq 0\right\} \\
& D_{i j \text {,mem }}^{\text {off }}:=\left\{(y, a, \theta) \in \mathbb{X}_{\mathrm{mem}} \mid a_{i j}=1, \Gamma_{i j}^{\mathrm{off}} \leq-\varepsilon, \theta_{i j} \leq 0\right\} .
\end{aligned}
$$

System (11) satisfies the hybrid basic conditions of [16, As. 6.5], in view of the definition of the flow and jump maps and the flow and jump sets. Then, from [16, Thm 6.30], it is (nominally) well-posed, namely its solutions satisfy a desirable sequential compactness property.

\section{B. Main stability result}

We establish here a $\mathcal{K} \mathcal{L}$-stability property for (11) generalizing the one established for (8) at the end of Section II. To 
this end, function $\omega_{0}$ in (9) is generalized to

$$
\omega(x):=\omega_{0}((y, a))+\sum_{(i, j) \in \mathcal{E}^{+}}\left(1-a_{i j}\right) \max \left\{0, \theta_{i j}\right\},
$$

for any $x \in \mathbb{X}_{\text {mem }}$ which incorporates the memory variable $\theta$. Since $\omega_{0}$ is continuous, then $\omega$ is continuous too on $\mathbb{X}_{\text {mem }}$. Our main result below ensures $\mathcal{K} \mathcal{L}$-stability of model (11) with respect to $\omega$, as well as properties of the hybrid time domains of its solutions. The proof of Theorem 1 is based on a novel hybrid Lyapunov function characterized in the next section.

Theorem 1: All maximal solutions to system (11) are complete and eventually continuous. For each maximal solution $x$, there exists $x^{\star} \in \mathbb{X}_{\text {mem }}$ such that $x(\mathfrak{t}, \mathfrak{j}) \rightarrow x^{\star}$ as $\mathfrak{t}+\mathfrak{j} \rightarrow \infty$. Moreover, system (11) is $\mathcal{K} \mathcal{L}$-stable with respect to $\omega$ in (12).

Since the second term in (12) is non-negative, the convergence to zero of $\omega(x)$ established in Theorem 1 immediately implies that $\omega_{0}((y, a)) \rightarrow 0$. As result, Theorem 1 ensures that opinions converge to clusters as time grows. In addition, $\left(1-a_{i j}\right) \max \left\{0, \theta_{i j}\right\}$ converges to zero for all $(i, j) \in \mathcal{E}^{+}$, namely the memory variable $\theta_{i j}$ associated to individuals belonging to different clusters is not positive. The asymptotic behavior of solutions is clarified in the following corollary, which is an immediate consequence of eventual continuity of solutions ( $a$ eventually settles to a clustering pattern) and convergence of solutions (opinions settle to constant values that coincide within each cluster because $\left.\omega_{0}((y, a)) \rightarrow 0\right)$.

Corollary 1: Each maximal solution of (11) converges to a clustering pattern with constant, equal opinions in each cluster.

\section{Lyapunov function and proof of Theorem 1}

Consider the following candidate Lyapunov function,

$$
U(x):=V(x)+\gamma \sum_{(i, j) \in \mathcal{E}^{+}}\left(1-a_{i j}\right) \max \left\{0, \theta_{i j}\right\},
$$

for each $x=(y, a, \theta) \in \mathbb{X}_{\text {mem }}, \gamma>0$ to be selected, and

$$
V(x):=\frac{1}{2} y^{\top} \Phi y=\frac{1}{4} \sum_{(i, j) \in \mathcal{V}^{2}} \varphi_{i j}\left(y_{i}-y_{j}\right)^{2} .
$$

The second equality above arises from the Dirichlet form [9, Prop. 1.9] and the definition of $\Phi$ after (4). Function $V$ was used in [12, eqn. (18)] and takes into account the opinions, while the second term in (13) accounts for the new dynamics $\theta$, paralleling the dynamic event-triggered control designs [15],[23]. We state below key properties of $U$.

Proposition 1: Given system (11), there exist $\gamma>0$ in (13) and $c_{1}, c_{2}, c_{F}, c_{J}>0$ such that the following holds.

(i) $U$ is locally Lipschitz on $\mathbb{X}_{\mathrm{mem}}$ and satisfies $c_{1} \omega(x) \leq$ $U(x) \leq c_{2} \omega(x)$ for all $x \in \mathbb{X}_{\mathrm{mem}}$.

(ii) For all $x \in C_{\mathrm{mem}}, U^{\circ}(x ; f(x)) \leq-c_{F} U(x)$.

(iii) For all $x \in D_{\text {mem }}$, and $v \in g(x), U(v)-U(x) \leq-c_{J}$.

Proof. We prove the three items one by one.

Proof of item $(i)$. Function $U$ is locally Lipschitz on $\mathbb{X}_{\mathrm{mem}}$ in view of its definition in (13). According to [12, eqn. (19)], there exist $\tilde{c}_{1}, \tilde{c}_{2}>0$ such that for any $(y, a) \in \mathbb{X}_{\text {inst }}$, $\tilde{c}_{1} \omega_{0}((y, a))^{2} \leq V(x) \leq \tilde{c}_{2} \omega_{0}((y, a))^{2}$. As a result, by the definition of $\omega$ in (12), we obtain the inequality in (i) with $c_{1}:=\min \left\{\tilde{c}_{1}, \gamma\right\}$ and $c_{2}:=\max \left\{\tilde{c}_{2}, \gamma\right\}$.
Proof of item (ii). Given any $x=(y, a, \theta) \in C_{\text {mem }}$, introduce the sets (below, for simplicity, the dependence on $x$ is sometimes omitted)

$$
\begin{aligned}
& \mathcal{E}_{>0}^{c}(x):=\left\{(i, j) \in \mathcal{E}^{+} \mid a_{i j}=0 \text { and } \theta_{i j}>0\right\}, \\
& \mathcal{E}_{=0}^{c}(x):=\left\{(i, j) \in \mathcal{E}^{+} \mid a_{i j}=0 \text { and } \theta_{i j}=0\right\}, \\
& \mathcal{E}_{\Gamma}^{c}(x):=\left\{(i, j) \in \mathcal{E}^{+} \mid \Gamma_{i j}^{\text {on }}(y, a) \geq 0\right\} .
\end{aligned}
$$

According to [21, Prop. 1.1], in view of (10), (11), (13) and the definition of $f_{\theta}$, we have that

$$
\begin{aligned}
U^{\circ}(x ; & f(x))=\langle\nabla V(x), f(x)\rangle \\
& +\gamma \sum_{(i, j) \in \mathcal{E}_{>0}^{c}}\left(-\beta_{i j} \theta_{i j}+\Gamma_{i j}^{\mathrm{on}}(y, a)\right) \\
& +\gamma \sum_{(i, j) \in \mathcal{E}_{=0}^{c}} \max \left\{0,-\beta_{i j} \theta_{i j}+\Gamma_{i j}^{\mathrm{on}}(y, a)\right\} \\
= & \langle\nabla V(x), f(x)\rangle+\gamma \sum_{(i, j) \in \mathcal{E}_{>0}^{c}}\left(-\beta_{i j} \theta_{i j}+\Gamma_{i j}^{\mathrm{on}}(y, a)\right) \\
& +\gamma \sum_{(i, j) \in\left(\mathcal{E}_{=0}^{c} \cap \mathcal{E}_{\Gamma}^{c}\right)} \Gamma_{i j}^{\mathrm{on}}(y, a) \\
= & \langle\nabla V(x), f(x)\rangle+\gamma \sum_{(i, j) \in \widehat{\mathcal{E}}}\left(-\beta_{i j} \theta_{i j}+\Gamma_{i j}^{\mathrm{on}}(y, a)\right)
\end{aligned}
$$

where $\left.\widehat{\mathcal{E}}(x):=\mathcal{E}_{>0}^{c}(x) \cup\left(\mathcal{E}_{=0}^{c}(x) \cap \mathcal{E}_{\Gamma}^{c}\right)(x)\right)$. Note that [12, eqn. (20)] holds because variables $(y, a)$ obey the same flow dynamics as in (8) and (11). Then $\langle\nabla V(x), f(x)\rangle \leq-\tilde{c}_{F} V(x)$ for some $\tilde{c}_{F}>0$. Consequently,

$$
U^{\circ}(x ; f(x)) \leq-\tilde{c}_{F} V(x)-\gamma \sum_{(i, j) \in \widehat{\mathcal{E}}} \beta_{i j} \theta_{i j}+\gamma \sum_{(i, j) \in \widehat{\mathcal{E}}} \Gamma_{i j}^{\mathrm{on}}(y, a) .
$$

The expression of $\Gamma_{i j}^{\text {on }}$ in (7), together with (14) yields

$$
\begin{aligned}
& \sum_{(i, j) \in \widehat{\mathcal{E}}} \Gamma_{i j}^{\mathrm{on}}(y, a)=\sum_{(i, j) \in \widehat{\mathcal{E}}}\left(-\left(1+\frac{\eta^{2}}{d_{i} d_{j}}\right)\left(y_{i}-y_{j}\right)^{2}\right. \\
& \left.+\sum_{\ell \neq i, \ell \neq j}\left(\left(d_{j}+1\right) \varphi_{i \ell}\left(y_{i}-y_{\ell}\right)^{2}+\left(d_{i}+1\right) \varphi_{j \ell}\left(y_{j}-y_{\ell}\right)^{2}\right)\right) \\
& \leq n \sum_{(i, j) \in \widehat{\mathcal{E}}_{\ell \neq i, \ell \neq j}}\left(\varphi_{i \ell}\left(y_{i}-y_{\ell}\right)^{2}+\varphi_{j \ell}\left(y_{j}-y_{\ell}\right)^{2}\right) \\
& \leq n \sum_{(i, \ell) \in \mathcal{V}^{2}} \varphi_{i \ell}\left(y_{i}-y_{\ell}\right)^{2}+n \sum_{(j, \ell) \in \mathcal{V}^{2}} \varphi_{j \ell}\left(y_{j}-y_{\ell}\right)^{2} \\
& =4 n V(x)+4 n V(x)=8 n V(x),
\end{aligned}
$$

which can be substituted in the preceding inequality to get

$$
U^{\circ}(x ; f(x)) \leq-\left(\tilde{c}_{F}-8 n \gamma\right) V(x)-\gamma \sum_{(i, j) \in \widehat{\mathcal{E}}} \beta_{i j} \theta_{i j} .
$$

Now, $\theta_{i j}=\left(1-a_{i j}\right) \max \left\{0, \theta_{i j}\right\}$ for $(i, j) \in \widehat{\mathcal{E}} \subset \mathcal{E}_{>0}^{c} \cup \mathcal{E}_{=0}^{c}$, and $\left(1-a_{i j}\right) \max \left\{0, \theta_{i j}\right\}=0$ for $(i, j) \in \mathcal{E}^{+} \backslash \widehat{\mathcal{E}}$, because either $a_{i j}=1$ or $\theta_{i j} \leq 0$ for those edges. Then,

$$
U^{\circ}(x ; f(x)) \leq-c_{F} U(x),
$$

where $c_{F}=\min \left\{\tilde{c}_{F}-8 n \gamma, \beta\right\}$ and $\beta:=\min _{(i, j) \in \mathcal{E}^{+}} \beta_{i j}>0$, which implies item (ii) with any $\gamma \in\left(0, \frac{\tilde{c}_{F}}{8 n}\right)$.

Proof of item (iii). From (11b), for each $x \in D_{\text {mem }}$ and $v \in$ $\overline{g(x) \text {, there exists }}(i, j) \in \mathcal{E}^{+}$such that $x \in D_{i j, \text { mem }}^{\text {on }} \cup D_{i j, \text { mem }}^{\text {off }}$, and $a^{+} \in g_{i j}(y, a)$. Since $D_{i j \text {,mem }}^{\text {on }}$ and $D_{i j, \text { mem }}^{\text {off }}$ are disjoint, then two cases may occur: case "on" and case "off" below.

Case "on": $x \in D_{i j \text {,mem. }}^{\text {on }}$. In this case, $a_{i j}=a_{j i}=0$, $\Gamma_{i j}^{\mathrm{on}}(y, a) \geq \varepsilon$ and $\theta_{i j} \geq 0$. Since link $(i, j)$ is activated at this jump, $a_{i j}^{+}=a_{j i}^{+}=1$ and from (5), (6), the other connectivity variables, as well as all the memory variables in view of (11), 
remain constant across the jump. Consequently,

$$
U(v)-U(x)=V(v)-V(x)-\gamma \max \left\{0, \theta_{i j}\right\} .
$$

Following the proof [12, Lemma 5], we get $V(v)-V(x)=$ $\frac{1}{2\left(d_{i}+1\right)\left(d_{j}+1\right)}\left(-\Gamma_{i j}^{\text {on }}(y, a)-\frac{\eta^{2}}{d_{i} d_{j}}\left(y_{i}-y_{j}\right)^{2}\right)$.

Thus, (19) yields

$$
U(v)-U(x) \leq \frac{1}{2 d_{i}^{+} d_{j}^{+}}\left(-\varepsilon-\frac{\eta^{2}}{d_{i} d_{j}}\left(y_{i}-y_{j}\right)^{2}\right),
$$

where $d_{i}^{+}=d_{i}+1$ and $d_{j}^{+}=d_{j}+1$. Taking $c_{J} \in\left(0, \frac{\varepsilon}{2 n^{2}}\right]$, characterizing the least possible decrease with $n$ agents, we prove item (iii) for the case "on".

Case "off": $x \in D_{i j \text {,mem. }}^{\text {off }}$. In this case, $a_{i j}=a_{j i}=1$, $\Gamma_{i j}^{\text {off }} \leq-\varepsilon$ and $\theta_{i j} \leq 0$. Link $(i, j)$ is deactivated at this jump and $a_{i j}^{+}=a_{j i}^{+}=0$. The other connectivity variables and the memory variables remain constant. Then (19) holds again and following again the proof of [12, Lemma 5], we deduce that $V(v)-V(x)=\frac{1}{2 d_{i} d_{j}}\left(\Gamma_{i j}^{\mathrm{off}}(y, a)-\frac{\eta^{2}}{d_{i} d_{j}}\left(y_{i}-y_{j}\right)^{2}\right)$. Thus, (19) yields

$$
U(v)-U(x) \leq \frac{1}{2 d_{i} d_{j}}\left(-\varepsilon-\frac{\eta^{2}}{d_{i} d_{j}}\left(y_{i}-y_{j}\right)^{2}\right) .
$$

Selecting $c_{J} \in\left(0, \frac{\varepsilon}{2 n^{2}}\right]$, item (iii) holds in case "off". Thus, item (iii) holds with $c_{J}:=\frac{\varepsilon}{2 n^{2}}$.

Proof of Theorem 1. To prove that maximal solutions to (11) are complete, we invoke [16, Prop. 6.10]. First, the viability condition is satisfied in view of the system definition. Secondly, $g\left(D_{\text {mem }}\right) \subset C_{\text {mem }} \cup D_{\text {mem }}$. Moreover, using $W(x)=$ $y^{\top} y$, we have $\langle\nabla W(x), f(x)\rangle=-2 y^{\top} \Phi y \leq 0$, therefore the $y$ components are bounded. Also the memory variables $\theta$ are bounded, because they are constant across jumps and the components of its flow map are exponentially stable filters with integrable inputs. Consequently, maximal solutions do not escape in finite time and [16, Prop. 6.10] establishes their completeness. Eventual continuity follows from the fact that the decrease of $U$ across jumps in item (iii) of Proposition 1 is constant at each jump and that $U$ does not increase on flows in item (ii) of Proposition 1, therefore any solution jumping forever would eventually lead to a negative $U(x)$, contradicting item (i) of Proposition 1. About convergence of solutions, state $a$ settles due to eventual continuity, $y$ settles too because $\langle\nabla W(x), f(x)\rangle=-2 y^{T} \Phi y=0$ and symmetry of $\Phi$ implies $-2 \dot{y}=\Phi y=0$, finally $\theta$ converges too because it is a linear filter with a converging input.

Let us now prove the $\mathcal{K} \mathcal{L}$ bound on the solutions. Since the conditions of Proposition 1 are analogous to those of [12, Lemma 5], we can proceed as in [12, eq. (23)] to obtain

$$
\begin{aligned}
& \omega(x(\mathfrak{t}, \mathfrak{j})) \leq \beta(\omega(x(0,0)), \mathfrak{t}, \mathfrak{j}) \\
& :=\frac{c_{2}}{c_{1}} \mathrm{e}^{c_{F} \cdot \mathfrak{t}}\left(1-\min \left\{1, \frac{c_{J}}{c_{2} \omega(x(0,0))}\right\}\right)^{\mathfrak{j}} \omega(x(0,0)),
\end{aligned}
$$

establishing a class $\mathcal{K} \mathcal{L} \mathcal{L}$ bound [3], which is easily transformed into a class $\mathcal{K} \mathcal{L}$ bound $\omega(x(\mathfrak{t}, \mathfrak{j})) \leq \bar{\beta}(\omega(x(0,0)), \mathfrak{t}+\mathfrak{j})$ constructing $\bar{\beta}$ from $\beta$ as in [3, Lemma 6.1].

\section{Simulations}

Consider $n=15$ agents, $\varepsilon=0.01$ and $\eta=3$ in (7). The initial topology is an Erdős-Rényi random graph, with probability $p$ of having an interconnection between each node pair, while the initial values of $y_{i}, i \in\{1, \ldots, n\}$ are selected randomly in the interval $[0,1]$. Simulation results for model (8) use the Matlab toolbox [24] and are reported in Fig. 1.
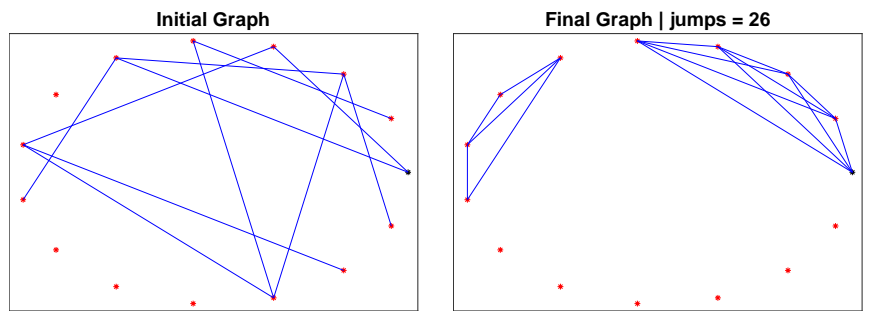

Fig. 1. Initial and final topologies for given $y(0,0)$, with $p=0.1$. Nodes have been sorted counterclockwise to clearly visualize the clusters appearing in the final topology.

We then study model (11), the impact of the choices of $\beta_{i j}$ and the initial values of $\theta_{i j}$ on the evolution of the opinions, for the same $y(0,0)$ as in Fig. 1. In particular, we take for $\beta_{i j}=\beta$ with $\beta \in\{0.1,50\}$, and $\theta_{i j}(0,0)=\theta^{o}$ with $\theta^{o} \in\{0,0.01,1\}$ for $a_{i j}(0,0)=1$ and $\theta^{o}=0$ otherwise, as well as the case where $\theta^{o}$ takes random values in $[-1,0)$, for all $(i, j) \in \mathcal{E}^{+}$. The final graphs are depicted in Fig. 2 and 3, showing that the opinions converge to fixed agreement values in each cluster, as stated in Corollary 1 . The communities arising from model (8) and (11) are generally different for any selection of initial conditions. The simulations show that the communities are significantly influenced by the novel memory variable. Only for $\theta^{\circ}=0$ the clusters in Fig. 3 are the same as those in Fig. 1. Indeed, even with a small value for $\theta^{\circ}$ in combination with a high value of $\beta$ leads to significantly different clusters compared to the ones in [12].

When comparing Fig. 2 and 3, we note that positive values of $\theta$ tend to preserve the existing initial interconnections, leading to larger clusters. This suggests that agents remember their mutual relationships with each other. On the other hand, negative values generate clusters made of fewer agents in general, see Fig. 2 compared to Fig. 3. The evolutions of $y$ and $\theta$ as functions of the continuous-time $t$ are depicted in Fig. 4 for $\beta=0.1$ and $\theta^{\circ}=1$. Two clusters appear as time grows, in agreement with the corresponding plot in Fig. 2. When agents $i$ and $j$ are in the same cluster, $\theta_{i j}$ converges to 0 just as $\Gamma_{i j}^{\text {off }}(y, a)$ does in this case. When agents $i$ and $j$ are not in the same cluster, $\theta_{i j}$ converges to the same values of $\Gamma_{i j}^{\mathrm{on}}(y, a) / \beta_{i j}$ in view of (10). This ratio, $\Gamma_{i j}^{\mathrm{on}}(y, a) / \beta_{i j}$, can take any constant value in $(-\infty, \varepsilon]$ in view of $(11 b)$. Hence, in some cases we have $\theta_{i j}(\mathfrak{t}, \mathfrak{j}) \rightarrow 0$ as $\mathfrak{t}+\mathfrak{j} \rightarrow \infty$ although agents $i$ and $j$ are not in the same cluster. That said, to distinguish agents from the same clusters, it is more relevant to monitor $\sigma_{i j}$ defined as:

$$
\sigma_{i j}:=\left(2 a_{i j}-1\right) \frac{\left|\theta_{i j}\right|}{\left|\left(1-a_{i j}\right) \Gamma_{i j}^{\mathrm{on}}(y, a)+a_{i j} \Gamma_{i j}^{\mathrm{off}}(y, a)\right|} .
$$



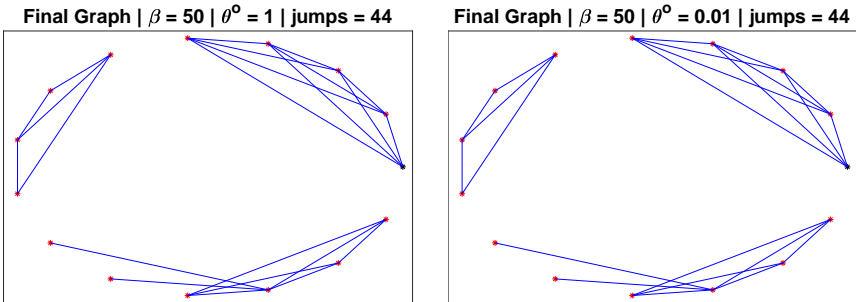

Final Graph $|\beta=0.1| \theta^{\circ}=1 \mid$ jumps $=35$
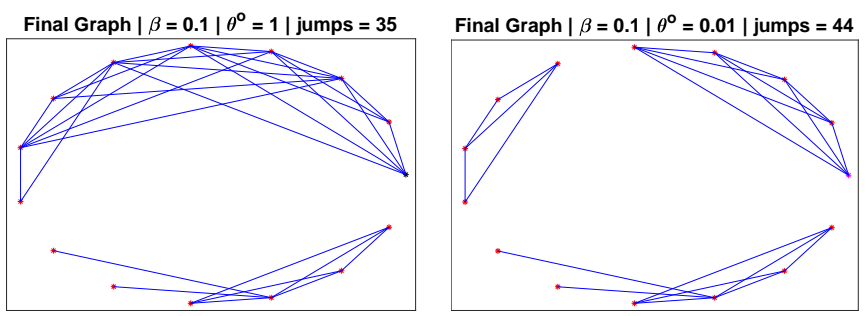

Fig. 2. Four different final topologies for different couples of $(\beta, \theta(0,0))$.
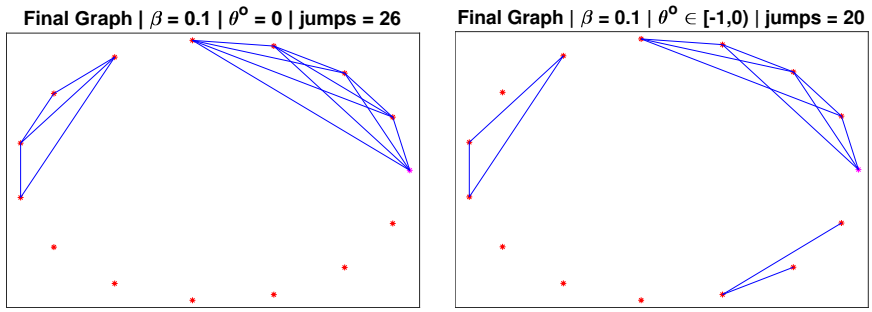

Fig. 3. Different final topologies for different, non positive $\theta(0,0)$.

At steady-state, $\sigma_{i j}$ either converges to $1 / \beta_{i j}$ if agents $i$ and $j$ belong to the same cluster, or to $-1 / \beta_{i j}$ otherwise. The influence of $\beta_{i j}$ is clear here: the smaller $\beta_{i j}$, the more important the past is and the bigger $\sigma_{i j}$, and vice versa.
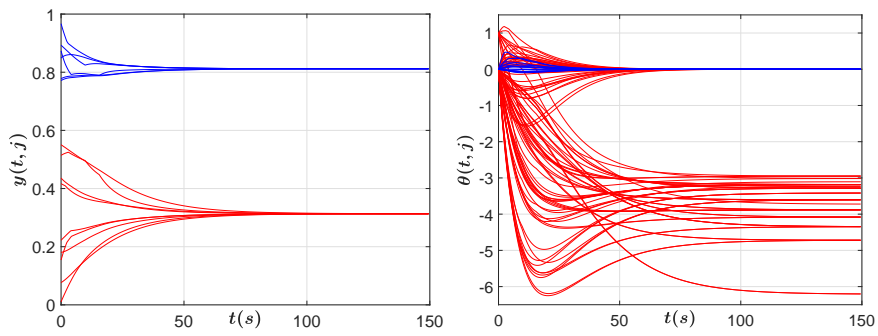

Fig. 4. Values of $y$ (left) and $\theta$ (right) for $\beta=0.1$ and $\theta^{\circ}=1$. Different colors have been used for different clusters in the final topology.

\section{CONCLUSIONS}

We have presented a hybrid model of opinion dynamics where the connectivity among individuals takes into account both the present and the past values of the opinions of the respective individuals. We believe that the idea of taking into account the past when defining connectivity in opinion dynamics is appealing and relevant, and that it has been largely unexplored so far. Further investigation on more complex opinion dynamics models could follow the path outlined in this work, exploiting the hybrid formalism, and hence, all the technical result available. Another direction to investigate resides in testing the proposed model on real world applications.

\section{ACKNOWLEDGEMENT}

The authors thank Alexis Navarian for his help in the initial stages of this work.

\section{REFERENCES}

[1] C. Altafini. Consensus problems on networks with antagonistic interactions. IEEE Trans. on Aut. Control, 58(4):935-946, 2013.

[2] P. Bolzern, P. Colaneri, and G. De Nicolao. Event-triggered real-time scheduling of stabilizing control tasks. Automatical, 100:219-230, 2019.

[3] A.R. Teel C. Cai and R. Goebel. Smooth Lyapunov functions for hybrid systems part I: Existence is equivalent to robustness. IEEE Trans. on Aut. Control, 52(7):1264-1277, 2007.

[4] F. Ceragioli and P. Frasca. Continuous-time consensus dynamics with quantized all-to-all communication. In Proceedings of European Control Conference, pages 1926-1931, 2015.

[5] N. R. Chowdhury, I.-C. Morărescu, S. Martin, and S. Srikant. Continuous opinions and discrete actions in social networks: a multi-agent system approach. In Proc. 55th IEEE C.D.C., pages 1739-1744, 2016.

[6] F. H. Clarke. Optimization and nonsmooth analysis. Classics in Applied Mathematics vol. 5, SIAM, Philadelphia, U.S.A, 1998.

[7] G. Deffuant, D. Neau, F. Amblard, and G. Weisbuch. Mixing beliefs among interacting agents. Advances in Complex Sys., 3:87-98, 2000.

[8] M. H. DeGroot. Reaching a consensus. Journal of the American Statistical Association, 69(345):118-121, 1974.

[9] F. Fagnani and P. Frasca. Introduction to averaging dynamics over networks. In Lecture notes in control and information sciences. Springer Nature, 2017.

[10] A. Flache, M. Mäs, T. Feliciani, E. Chattoe-Brown, G. Deffuant, S. Huet, and J. Lorenz. Models of social influence: towards the next frontiers. $J$. of Artificial Soc. and Social Simulation, 20(4):2, 2017.

[11] P. Frasca. Continuous-time quantized consensus: Convergence of Krasovskii solutions. Systems \& Control Letters, 61(2):273-278, 2012.

[12] P. Frasca, S. Tarbouriech, and L. Zaccarian. Hybrid models of opinion dynamics with opinion-dependent connectivity. Automatica, 100:153$161,2019$.

[13] N. E. Friedkin and E. C. Johnsen. Social influence and opinions. Journal of Mathematical Sociology, 15:193-206, 1990.

[14] S. Galam and S. Moscovici. Towards a theory of collective phenomena: Consensus and attitude changes in groups. European Journal of Social Psychology, 21(1):49-74, 1991.

[15] A. Girard. Dynamic triggering mechanisms for event-triggered control. IEEE Trans. on Aut. Control, 60(7):1992-1997, 2015.

[16] R. Goebel, R.G. Sanfelice, and A.R. Teel. Hybrid dynamical systems. Princeton University Press, Princeton, U.S.A., 2012.

[17] R. Hegselmann and U. Krause. Opinion dynamics and bounded confidence models, analysis, and simulation. Journal of Artificial Societies and Social Simulation, 5(3), 2002.

[18] C. Vande Kerckhove, S. Martin, P. Gend, P. J. Rentfrow, J. M. Hendrickx, and V. D. Blondel. Modelling influence and opinion evolution in online collective behaviour. PLOS ONE, 11(6):1-25, 2016.

[19] A.C.R. Martins. Continuous opinions and discrete actions in opinion dynamics problems. Int. Jour. of Modern Physics C, 19(4):617-625, 2008.

[20] I.-C. Morărescu and A. Girard. Opinion dynamics with decaying confidence: Application to community detection in graphs. IEEE Trans. on Aut. Control, 56(8): 1862 - 1873, 2011.

[21] D. Nešić and A. Teel. A Lyapunov-based small-gain theorem for hybrid ISS systems. In 47th IEEE C.D.C., pages 3380-3385, 2008.

[22] S. E. Parsegov, A. V. Proskurnikov amd R. Tempo, and N. E. Friedkin. Novel multidimensional models of opinion dynamics in social networks. IEEE Trans. on Aut. Control, 62(5):2270-2285, 2016.

[23] C. De Persis and R. Postoyan. A Lyapunov redesign of coordination algorithms for cyber-physical systems. IEEE Trans. on Aut. Control, 62(2):808-823, 2017.

[24] R.G. Sanfelice, D.A. Copp, and P. Nanez. A toolbox for simulation of hybrid systems in matlab/simulink: hybrid equations (hyeq) toolbox. In HSCC, page 101-106, 2013.

[25] P. Tabuada. Event-triggered real-time scheduling of stabilizing control tasks. IEEE Trans. on Aut. Control, 52(9):1680-1685, 2007.

[26] A.R. Teel and L. Praly. A smooth Lyapunov function from a class- $\mathcal{K} \mathcal{L}$ estimate involving two positive semidefinite functions. ESAIM: Control, Optimisation and Calculus of Variations, 5(1):313-367, 2000.

[27] V.S. Varma and I.-C. Morărescu. Modeling stochastic dynamics of agents with multi-leveled opinions and binary actions. In 55th IEEE C.D.C., pages 1064-1069, 2017. 